\title{
Crossing Methods and Cultivation Conditions for Rapid Production of Segregating Populations in Three Grain Amaranth Species
}

\author{
Markus G. Stetter*, Leo Zeitler, Adrian Steinhaus, Karoline Kroener, Michelle Biljecki and \\ Karl J. Schmid
}

Institute of Plant Breeding, Seed Science and Population Genetics, University of Hohenheim, Stuttgart, Germany

\section{OPEN ACCESS}

Edited by:

Antonio M. De Ron, Spanish National Research Council (CSIC) - Misión Biológica de Galicia (MBG), Spain

Reviewed by: Johann Vollmann, University of Natural Resources and

Life Sciences, Austria

Peter Jeff Maughan, Brigham Young University, USA David M. Brenner,

lowa State University, USA

*Correspondence:

Markus G. Stetter

markus.stetter@uni-hohenheim.de

Specialty section: This article was submitted to

Crop Science and Horticulture, a section of the journa Frontiers in Plant Science

Received: 20 April 2016 Accepted: 25 May 2016 Published: 07 June 2016

Citation:

Stetter MG, Zeitler L, Steinhaus A, Kroener K, Biljecki M and Schmid KJ (2016) Crossing Methods and

Cultivation Conditions for Rapid Production of Segregating Populations in Three Grain Amaranth

Species. Front. Plant Sci. 7:816 doi: $10.3389 / \mathrm{fp} / \mathrm{s} .2016 .00816$
Grain amaranths (Amaranthus spp.) have been cultivated for thousands of years in Central and South America. Their grains are of high nutritional value, but the low yield needs to be increased by selection of superior genotypes from genetically diverse breeding populations. Amaranths are adapted to harsh conditions and can be cultivated on marginal lands although little is known about their physiology. The development of controlled growing conditions and efficient crossing methods is important for research on and improvement of this ancient crop. Grain amaranth was domesticated in the Americas and is highly self-fertilizing with a large inflorescence consisting of thousands of very small flowers. We evaluated three different crossing methods (open pollination, hot water emasculation and hand emasculation) for their efficiency in amaranth and validated them with genetic markers. We identified cultivation conditions that allow an easy control of flowering time by day length manipulation and achieved flowering times of 4 weeks and generation times of 2 months. All three different crossing methods successfully produced hybrid $F_{1}$ offspring, but with different success rates. Open pollination had the lowest $(10 \%)$ and hand emasculation the highest success rate (74\%). Hot water emasculation showed an intermediate success rate (26\%) with a maximum of $94 \%$ success. It is simple to perform and suitable for a more large-scale production of hybrids. We further evaluated 11 single nucleotide polymorphism (SNP) markers and found that they were sufficient to validate all crosses of the genotypes used in this study for intra- and interspecific hybridizations. Despite its very small flowers, crosses in amaranth can be carried out efficiently and evaluated with inexpensive SNP markers. Suitable growth conditions strongly reduce the generation time and allow the control of plant height, flowering time, and seed production. In combination, this enables the rapid production of segregating populations which makes amaranth an attractive model for basic plant research but also facilitates further the improvement of this ancient crop by plant breeding.

Keywords: amaranth, hybridization, hot water emasculation, hand emasculation, genetic resources, marker assisted breeding 


\section{INTRODUCTION}

Ancient crops from the Americas such as quinoa (Chenopodium quinoa willd.) or amaranth (Amaranthus spp. L.) are a valuable addition to the human diet because of their high nutritional value. These pseudocereals have a high protein content and are rich in lysine and other essential amino acids that are limited in other grains (Vega-Gálvez et al., 2010; Rastogi and Shukla, 2013). In addition, these crops are well adapted to harsh environmental conditions and are therefore suitable for cultivation on marginal soils. Their yields are significantly lower than those of major crops due to a lack of plant breeding (Reta Alemayehu et al., 2015), but the presence of a high genetic and phenotypic diversity in these species indicates an excellent potential for breeding and variety development (Brenner et al., 2010).

Grain amaranth originated from Central and South America, where it was of great importance in pre-columbian agriculture until its cultivation strongly declined after the Spanish conquest (Sauer, 1967; Kauffman and Weber, 1990; Brenner et al., 2010). Three species of Amaranthus are cultivated for grain production: A. caudatus L., A. cruentus L., and A. hypochondriacus L. Amaranth expresses the $\mathrm{C}_{4}$ carbon cycle, which is more common in grasses but rare in dicots. Despite a high genetic diversity (Stetter et al., 2015), breeding efforts in amaranth so far were limited to the selection of suitable genotypes from landraces. Amaranth is mainly self-pollinating and has numerous intricate flowers, which make crosses more difficult than in other crops. The ability to efficiently carry out crosses is an important requirement for plant research to understand genetic basis of relevant traits (Moose and Mumm, 2008; Olsen and Wendel, 2013). Crosses are equally important for plant breeding and are used to generate new genetic variation and to introgress exotic material into breeding populations.

In many crops, hybrid varieties are characterized by strongly increased yields (Duvick, 2001). The application of hybrid breeding in amaranth is also very promising, because a midparent heterosis of up to $88 \%$ has been reported (Lehmann et al., 1991). The ability to conduct crosses on a large scale with little effort is of central importance for the development and production of hybrid crop varieties. To use this potential in minor crops, an improvement of crossing methods is essential (Veerappan et al., 2014).

Several approaches for hybrid production are available, but for all methods the key step is to prevent self-fertilization by the male parent. This is either by using appropriate genetic self-incompatibility systems or by mechanical and chemical treatments that lead to male sterility. In several species, cytoplasmatic male sterility (CMS) systems prevent selfing of the female crossing partner (Laser and Lersten, 1972). To use CMS systems for breeding male sterile female parent and male parents with restorer genes are needed to allow seed production in the hybrid progeny. Additionally, a maintainer line is needed that allows multiplying the male sterile line without loosing the CMS. Male sterility has been reported in A. hypochondriacus but is not yet developed sufficiently to be used for breeding (Peters and Jain, 1987). Mechanical emasculation methods are efficient if the male and female flower are well separated on the plant (e.g., as in maize) because then male flowers can be removed without interfering with the female inflorescence. In other crops like tomato and Medicago, anthers are removed before pollen shedding (Veerappan et al., 2014). Another physical method is the heat treatment of the flowers of the female parent to destroy the pollen, for example by a hot water treatment. Here, the temperature is crucial, as differences by few degrees can influence the efficiency of the emasculation (Mukasa et al., 2007; GarcíaYzaguirre and Carreres, 2008; Otsuka et al., 2010). Chemical gametocides are used in hermaphrodite crops for which no CMS systems are available or are too costly, for example in wheat (Dotlacil and Apltauerová, 1978). The grain amaranth species have male and female flowers on the same inflorescence where several female flowers are arranged circularly around a male flower (Figure 1). The flowers are less than $1 \mathrm{~mm}$ in diameter, which makes mechanical emasculation difficult. For this reason other emasculation methods such as a hot water treatment may be more efficient.

Frequently, crossing methods are not completely reliable and require the validation of progeny. Phenotypic traits with a dominant-recessive inheritance can be used to identify successful crosses. In amaranth, traits such as seed or leaf color differ between genotypes and are available for validation (Kulakow et al., 1985). For phenotypic traits to be useful, however, parents need to differ in at least one trait and the male parent needs to express the dominant allele. In contrast, molecular markers allow an efficient and early evaluation of crosses without restricting the combination of parents, and cost-efficient PCR-based marker systems are available for this purpose (Maughan et al., 2011).

For model plants it is important to take specific requirements of development into account. Amaranth shows a strong photoperiod sensitivity and starts to flower under short day conditions (Brenner et al., 2010). A single plant has the potential

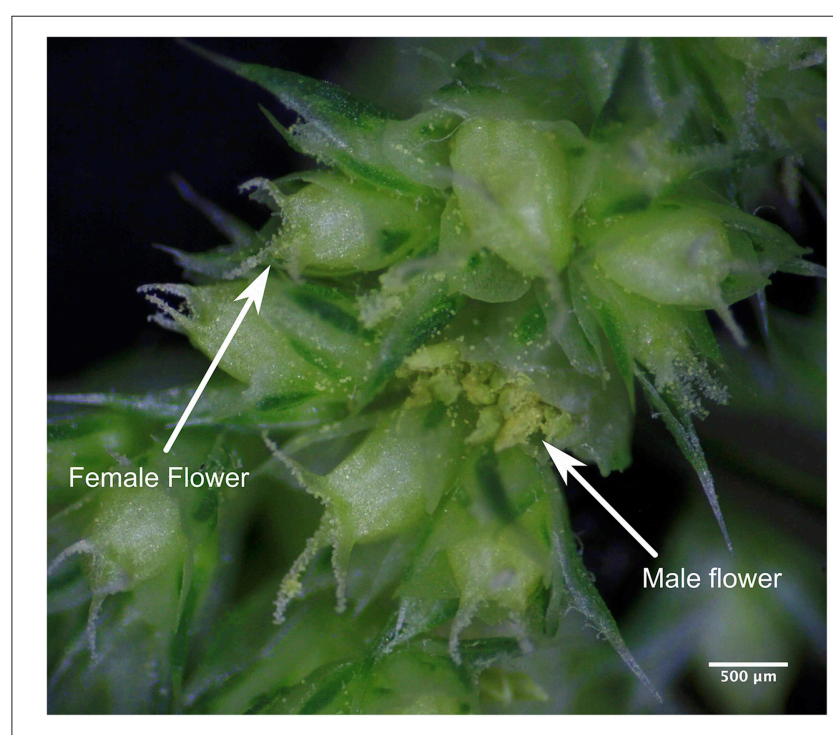

FIGURE 1 | Flower morphology. Inflorescence of $A$. caudatus consisting of flower clusters in which a male flower in the center is surrounded by several female flowers. 
to produce several thousands of seeds and can therefore produce large populations. However, under field conditions amaranth plants are usually tall and require a significant amount of space for cultivation. If flowering time, plant size and seed production can be controlled in climate chambers and greenhouses, an efficient propagation of the plant may be possible.

In the work presented here we study the efficiency of three different crossing methods and describe environmental conditions in a controlled environment (growth chamber) to achieve efficient and rapid generation of progeny for genetic studies. We suggest a method for hybrid identification with cost efficient PCR- based markers. Subsequently, we apply our method to three species of amaranth to evaluate its potential for the wider application to species within the genus Amaranthus.

\section{METHODS}

\subsection{Plant Material and Growth Conditions}

The amaranth accessions for testing the three crossing methods were selected to comprise accessions with green seedlings as female parent and accessions with red seedlings as male parent. Additionally, amaranth varieties were used to verify hybridization and the use of genetic markers (Table 1 and Table S2). Single seeds were planted in $7 \times 7 \mathrm{~cm}$ pots in standard gardening soil. Plants were grown for 2 weeks under long day conditions (Table 2) before transferring them step-wise in weekly intervals to short day conditions (Table 2). This helped to synchronize flowering of different genotypes and spread workload for performing the crosses.

\subsection{Crossing Methods}

We evaluated three different methods for crossing wild (A. hybr. and $A$. hybridus) and cultivated species of amaranth (Figure 2 and Table 1). The first method was open pollination by fixing the flowers of the female and male parent to each other and protecting them with a pollen proof bag (Sealed Air, Germany) from cross pollination by other plants. The second method was a warm water treatment of the inflorescence during flower initiation of the first emerging flowers (García-Yzaguirre and Carreres, 2008). Female flowers were dipped into a water bath of $45^{\circ} \mathrm{C}$ warm water for $10 \mathrm{~min}$ to emasculate the male flowers before proceeding as in the first method. The water treatment was repeated after 7 days. The third method was hand emasculation. For this approach, female flowers that were already open and all male flowers were removed from the inflorescence. The tip of the inflorescence was also removed to prevent the emergence of new flowers. The emasculation was repeated after 7 days and any flowers that developed later were removed. For all three methods plants were shaken daily to increase pollen dispersal and to assure cross-fertilization.

\subsection{Success Evaluation and Statistical Analysis}

Seeds of the female parent were harvested 4 weeks after crossing. For each cross 50 seeds were counted and planted in pots. Seedling color evaluation was performed 2 weeks after planting by counting green and red seedlings. The success rate was the

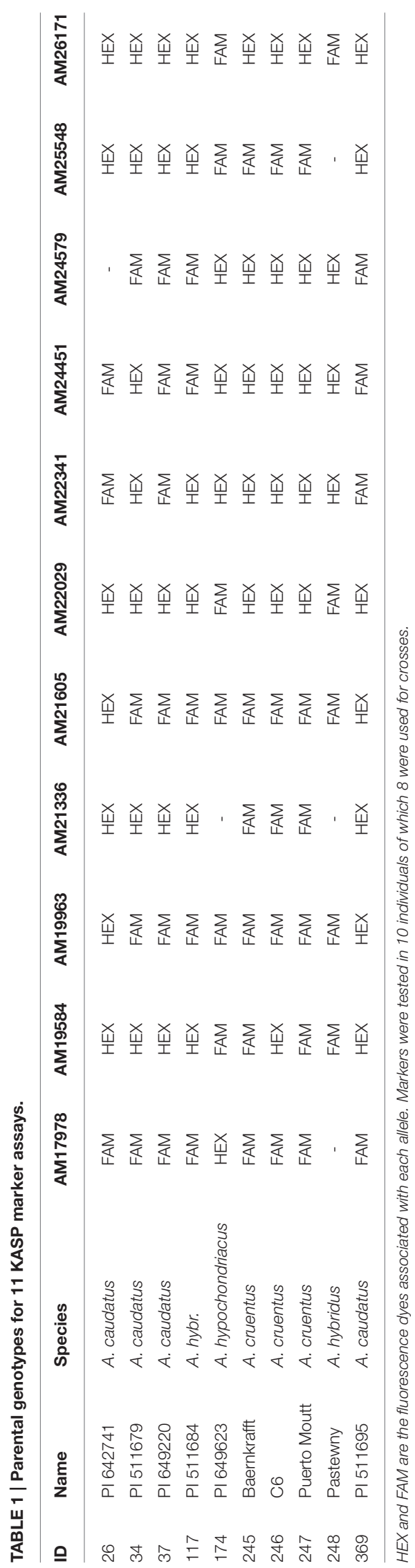


ratio between red and green offspring. Data analysis to test the differences between methods and between crossing types was conducted with a Generalized Linear Model (GLM) with binomial variance and a logit link function that included the crossing method $(\alpha)$, the crossing type ( $\beta$, Intra- and interspecific) and the interaction as factors:

$$
\operatorname{logit}\left(\mu_{i j}\right)=\log \left(\frac{\mu_{i j}}{1-\mu_{i j}}\right)=\eta_{i j}=\mu+\alpha_{i}+\beta_{j}+(\alpha \beta)_{i j} .
$$

The calculation was done with the $\mathrm{R}$ statistical package version 3.2.0 using the stats library.

\subsection{DNA Extraction}

For genotyping the DNA was extracted with EconoSpin columns (Epoch Life Science Inc.) using 1\% CTAB extraction buffer (Saghai-Maroof et al., 1984). Dry leaf samples were homogenized and incubated for $2 \mathrm{~h}$ at $50^{\circ} \mathrm{C}$ in $400 \mu \mathrm{l} 1 \% \mathrm{CTAB}$ extraction buffer and $4 \mu \mathrm{l}$ Proteinase K. After addition of $300 \mu \mathrm{l}$ Ammonium acetate $(7.5 \mathrm{M})$ and $300 \mu \mathrm{l}$ Ethanol (96\%), the samples were centrifuged for $1 \mathrm{~min}$ at full speed. Then $800 \mu \mathrm{l}$ of the supernatant were transferred on a EconoSpinÂ placed in the collection tube and centrifuged for $1 \mathrm{~min}$. The flow through was discarded. The columns were washed twice with wash buffers from Saghai-Maroof et al. (1984) before eluting DNA twice with $50 \mu \mathrm{l}$ Tris- $\mathrm{HCl}(10 \mathrm{mM}, \mathrm{pH} 8)$.

\subsection{Evaluation of Genetic Markers}

Eleven KASP assays (LGC Berlin/Germany) were selected from Maughan et al. (2011) to validate crosses (Table S1). The assays were prepared with $5 \mu \mathrm{l}$ DNA $(10 \mathrm{ng} / \mu \mathrm{l})$ and $5 \mu$ l genotyping mix and run on the LightCycler 480 Instrument II (Roche Life Science) with standard settings as given by the KASP manual (LGC Berlin/Germany) and analyzed using the LightCycler 480

TABLE 2 | Growth conditions.

\begin{tabular}{lcccc}
\hline & Day length & Light intensity & Temp day & Temp night \\
\hline Long day & 16 & $150 \mathrm{mmol}$ & $35^{\circ} \mathrm{C}$ & $30^{\circ} \mathrm{C}$ \\
Short day & 8 & $150 \mathrm{mmol}$ & $30^{\circ} \mathrm{C}$ & $25^{\circ} \mathrm{C}$ \\
\hline
\end{tabular}

Parameters for amaranth in growth chamber for long and short day conditions.
Software. First, parental lines were evaluated to find polymorphic markers for each of the crosses. Later, these markers were used to validate the crosses. For a proof of concept we genotyped offspring that were evaluated before by their seedling color. Both offspring with green (selfed plants) and red (hybrids) were genotyped.

\subsection{Additional Hybrid Production}

The previously evaluated hand emasculation method was used to produce additional hybrids. Plants were grown as described above, but crossing partners were not restricted to different seedling colors. The success of the crosses was validated with SNP markers.

\section{RESULTS AND DISCUSSION}

\subsection{Cultivation and Life Cycle}

In the field the generation time of the three grain amaranth species is approximately 6 months and leads to very tall plants with thousands of flowers. To reduce the generation time, plant height and number of flowers, we cultivated the plants under short day conditions $(8 \mathrm{~h})$ and high temperature $\left(30^{\circ} \mathrm{C}\right)$ which both induced early flowering 4 weeks after planting. Additionally, we controlled the initiation of flowering by transferring plants from long day $\left(16 \mathrm{~h}, 35^{\circ} \mathrm{C}\right)$ conditions to short day conditions. Under long day conditions the plants displayed strong vegetative growth and did not flower within 10 weeks after planting, but started flowering approximately 14 days after a transfer to short day conditions. The step-wise transfer of plants from long to short-day conditions allows the production of plants in different flowering stages, which greatly facilitates synchronous flowering for crosses between genotypes that differ in their flowering time. This treatment is further useful to produce male parents that are able to shed large amounts of pollen when females parents start flowering. As soon as 4 weeks after flowering, mature seeds could be harvested. By employing these treatments, very short generation times can be achieved that allow up to six generations per year, which is comparable to the model plant Arabidopsis thaliana. In addition, plant height and seed number per plant can be controlled by adjusting growth conditions. Long day conditions lead to more vegetative growth, later flowering and more seeds, while short day conditions result in small early

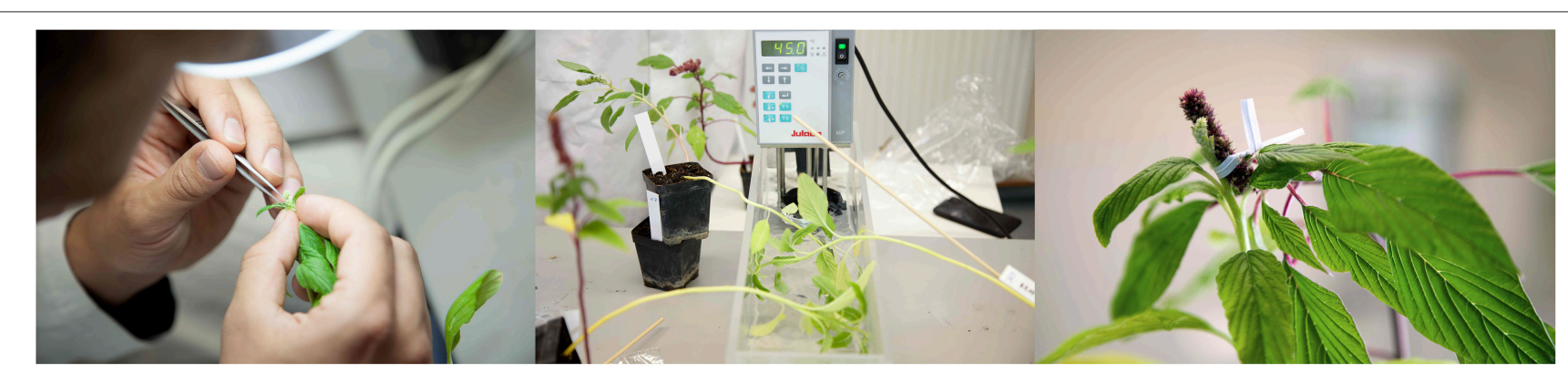

FIGURE 2 | Crossing methods. Three crossing methods: (A) Hand emasculation by removal of male flowers from female plant. (B) Hot water emasculation by 10 min treatment with $45^{\circ} \mathrm{C}$ water bath. (C) Fixing male and female flower to each other for better pollen transfer. 
TABLE 3 | Success rate of different crossing methods.

\begin{tabular}{|c|c|c|c|c|c|c|}
\hline & Type & $N$ & Mean (\%) & SD (\%) & Minimum (\%) & Maximum (\%) \\
\hline \multirow[t]{3}{*}{ Open pollination } & & 7 & $10^{C}$ & 5 & 4 & 18 \\
\hline & Intra-specific & 3 & 11 & 3 & 8 & 14 \\
\hline & Inter-specific & 4 & 10 & 6 & 4 & 18 \\
\hline \multirow[t]{3}{*}{ Heat treatment } & & 8 & $26^{b}$ & 35 & 0 & 94 \\
\hline & Intra-specific & 4 & 26 & 45 & 0 & 94 \\
\hline & Inter-specific & 4 & 27 & 27 & 0 & 57 \\
\hline \multirow[t]{3}{*}{ Hand emasculation } & & 11 & $74^{a}$ & 29 & 17 & 100 \\
\hline & Intra-specific & 4 & 80 & 20 & 50 & 94 \\
\hline & Inter-specific & 7 & 71 & 34 & 17 & 100 \\
\hline
\end{tabular}

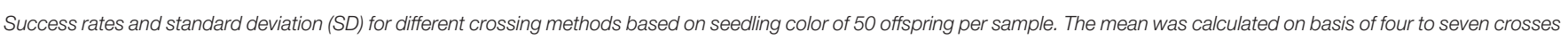
( $N$ ) per method and crossing type. Intra-specific crosses were performed with A. caudatus (PI $511679 \times$ PI 649220) and inter-specific crosses between A. caudatus (PI 511679) and A.

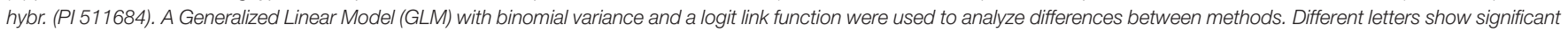
differences between methods. There was no significant difference between intra- and inter- specific crosses.

flowers. This is useful for different applications, because crosses require only few flowers, whereas the resulting $\mathrm{F}_{1}$ plants should produce larger amounts of seeds (e.g., for creating mapping populations).

\subsection{Crossing Methods}

We compared three crossing methods that included open pollination, hot water emasculation and hand emasculation. All three methods produced successful crosses, but the success rates and variances differed strongly between the methods (Table 3). The amount of seeds produced did not substantially differ between methods and the mother plants produced between 100 and 200 seeds. Open pollination between two plants under a single bag without emasculation of the female parent led to a mean success rate of $10 \%$ with a standard deviation (s.d.) of 0.05 . The hot water treatment of the female parent led to a significantly increased success rate of $26 \%$ but with a very high deviation (s.d. $=0.35$ ) and a minimal success rate of $0 \%$. However, the maximal success after hot water treatment was $94 \%$, which shows that the method has a high potential if the key conditions for a successful application can be identified. We sterilized flowers at $45^{\circ} \mathrm{C}$ and an adaptation of temperature may contribute to a higher rate of success. In other species (e.g., Acacia, buckwheat and rice), different temperatures change the efficiency of emasculation (Mukasa et al., 2007; García-Yzaguirre and Carreres, 2008; Otsuka et al., 2010). A temperature of $45^{\circ} \mathrm{C}$ for emasculation is rather high compared to other crops (GarcíaYzaguirre and Carreres, 2008), but not too high because the amaranth plants still set seeds after this treatment and a further optimization may be achieved by varying the length of the heat treatment. Overall, hot water emasculation works with amaranth and, if it can be further improved, is suitable for application in the field to large numbers of plants.

The most elaborated and time consuming method we evaluated was hand emasculation (Figure 3). The mean success rate of $74 \%$ was the highest of the three methods and the deviation (s.d. $=0.29$ ) was lower than of the heat treatment.

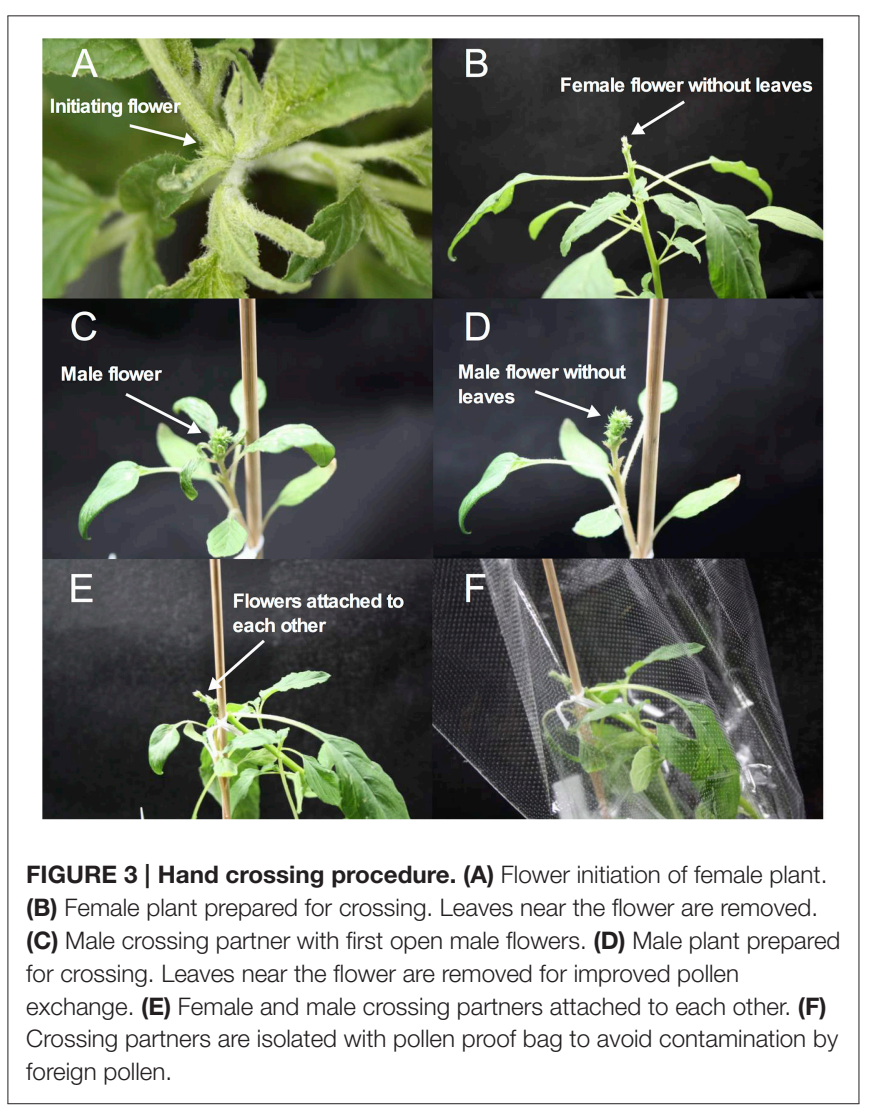

The minimum success was comparable to free pollination, but the maximum success was up to $100 \%$. Hand emasculation is difficult because amaranth has many small flowers and each male flower sheds enough pollen to pollinate a whole plant. Therefore it is critical to remove all male flowers from the female parent before flower dehiscence. The deviation can be decreased by keeping only few flower clusters per plant. We also tested whether intra- and inter-specific crosses are different in their 
efficiency, but there was no significant difference between intraand inter-specific crosses (Table 3). This shows that inter-specific hybridization is possible, but as the two species are closely related this might not be the case for distant member of the Amaranthus genus.

The comparison of the three methods shows that open pollination had low success rates, whereas heat treatment can be an effective and simple method for crosses if many seeds are required and simple morphological markers are available for the evaluation of offspring. Hand emasculation by well trained personnel shows the best performance. Since amaranth plants may produce thousands of seeds, a single successful cross can produce large $\mathrm{F}_{2}$ populations, and the number of hand crosses needed can be kept low, which decreases the work load of the method substantially and makes it suitable for large projects.

\subsection{Genetic Markers for Hybrid Identification}

Since no crossing method provides a $100 \%$ success rates, unsuccessful crosses have to be excluded in early stages. Furthermore, crossing partners should not be limited by phenotypic differences in certain traits (e.g., different seedling color), but all possible combinations parents should be available. We therefore evaluated all accessions used in this study with 11 PCR-based SNP markers. The markers were the most polymorphic from a set of 411 KASP markers from Maughan et al. (2011). Each marker was polymorphic between at least two lines and each cross segregated at least for one marker (Table 1). After evaluating the parental lines, we selected suitable markers to evaluate crosses.

First, we investigated progeny which had already been evaluated by their seedling color, because we expected green
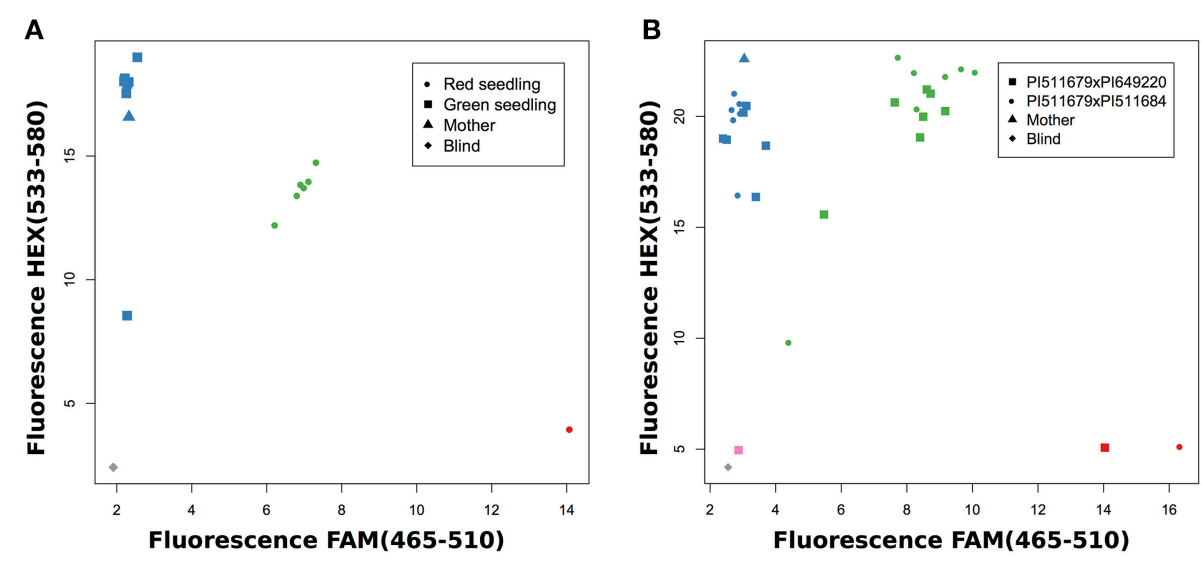

FIGURE 4 | SNP genotyping for known crosses. (A) Validation of PI511679xPI649220 with AM22341 and comparison with seedling color. (B) Validation of two crosses with AM24451.

TABLE 4 | Crosses of different amaranth varieties by hand emasculation and evaluation of success rates with SNP markers.

\begin{tabular}{|c|c|c|c|c|c|c|c|}
\hline & ID (Mother) & ID (Father) & Marker & Genotyped & Selfings & Successful crosses & Failed assay \\
\hline 1 & 34 & 245 & AM19584 & 7 & 0 & 7 & 0 \\
\hline 2 & 34 & 245 & AM19584 & 6 & 2 & 2 & 2 \\
\hline 3 & 34 & 245 & AM19584 & 7 & 2 & 4 & 1 \\
\hline 4 & 34 & 248 & AM19584 & 7 & 2 & 4 & 1 \\
\hline 5 & 34 & 248 & AM19584 & 7 & 0 & 6 & 1 \\
\hline 6 & 34 & 248 & AM19584 & 7 & 0 & 4 & 3 \\
\hline 7 & 245 & 26 & AM19584 & 6 & 4 & 2 & 0 \\
\hline 8 & 245 & 26 & AM19584 & 8 & 2 & 3 & 3 \\
\hline 9 & 247 & 248 & AM22029 & 10 & 1 & 9 & 0 \\
\hline 10 & 247 & 248 & AM22029 & 9 & 9 & 0 & 0 \\
\hline 11 & 248 & 245 & AM22029 & 6 & 6 & 0 & 0 \\
\hline 12 & 248 & 245 & AM22029 & 8 & 5 & 0 & 3 \\
\hline 13 & 248 & 245 & AM22029 & 7 & 4 & 0 & 3 \\
\hline
\end{tabular}

All crosses are interspecific crosses between the three grain amaranths and/or their putative ancestors. The ID of mothers and fathers corresponds to Table 1. 
seedlings to be homozygous for the maternal allele since the green allele is recessive, and red seedlings to be heterozygous. For example, the application of marker AM22341 in a cross of PI511679 $\times$ PI649220 showed that green seedlings were homozygous for the allele of parent PI511679 and red seedlings were heterozygous for both parental alleles (Figure 4A). Frequently, the same marker can be used in several crosses, which allows the evaluation of more than one cross simultaneously (Figure 4B). This strongly decreases the work load and the cost of the evaluation. When working with homozygous parental lines a single maker is sufficient to validate successful crosses.

To test the effectiveness of the hand crossing method and the validation with genetic markers, we produced hybrids between amaranth genotypes from different species and validated them with the marker system. Although not all crosses produced hybrids, for most crosses the number of hybrids produced was high and less than 10 offspring had to be evaluated per cross (Table 4).

\section{CONCLUSIONS}

Ancient and underutilized crops greatly benefit from the ongoing revolution in genomics. However, to utilize this information for the improvement of minor crops, efficient crossing methods which are the basis of breeding programs need to be established. We developed crossing methods and genetic markers for hybrid identification in amaranth and showed that these can be used for crosses within and between species. We further showed that the life cycle and plant size of amaranth can be reduced substantially when light and temperature conditions are adapted. For genetic and physiological studies a short generation time is advantageous, which is a common characteristic of model organisms for basic research. Under the conditions described here, generation times as short as those of $A$. thaliana are possible (Meyerowitz and Pruitt, 1985). Additionally, the amount of seeds can be controlled, which allows the production of large offspring

\section{REFERENCES}

Brenner, D. M., Baltensperger, D. D., Kulakow, P. A., Lehmann, J. W., Myers, R. L., Slabbert, M. M., et al. (2010). "Genetic resources and breeding of Amaranthus," in Plant Breeding Reviews, Vol. 19 (New York, NY: John Wiley \& Sons, Inc.), 227-285. doi: 10.1002/9780470650172.ch7

Clouse, J. W., Adhikary, D., Page, J. T., Ramaraj, T., Deyholos, M. K., Udall, J. A., et al. (2016). The amaranth genome: genome, transcriptome, and physical map assembly. Plant Gen. 9, 1-14. doi: 10.3835/plantgenome2015.07.0062

Dotlacil, L., and Apltauerová, M. (1978). Pollen sterility induced by ethrel and its utilization in hybridization of wheat. Euphytica 27, 353-360.

Duvick, D. N. (2001). Biotechnology in the 1930s: the development of hybrid maize. Nat. Rev. Genetics 2, 69-74. doi: 10.1038/35047587

García-Yzaguirre, A., and Carreres, R. (2008). Efficiency of different hybridization methods in single crosses of rice for pure line breeding. Spanish J. Agricult. Res. 6, 395-400. doi: 10.5424/sjar/2008063-332

Kauffman, C. S., and Weber, L. E. (1990). "Grain Amaranth," in Advances in New Crops, eds J. Janick and J. E. Simon (Portland: Timber Press), 127-139.

Kulakow, P., Hauptli, H., and Jain, S. (1985). Genetics of grain amaranths I. Mendelian analysis of six color characteristics. J. Heredity 76, 27-30. populations for genetic mapping. Furthermore, amaranth has a relatively small genome (500 $\mathrm{Mbp}$ ) with a reference sequence, and a large number of genotyped genebank accessions are available (Stetter et al., 2015; Clouse et al., 2016). Taken together, these resources and the possibility of interspecific crosses make the grain amaranth species a very suitable model organism for studying fundamental processes such as adaptation, speciation, heterosis, $\mathrm{C}_{4}$ photosynthetic metabolism, or domestication. The ability to conduct crosses from genetically diverse material facilitates the establishment of advanced breeding programs and the selection of improved genotypes using current breeding methods such as genomic selection will improve the value of this minor crop for agricultural production.

\section{AUTHOR CONTRIBUTIONS}

KS and MS designed the experiments. LZ, AS, KK, and MB performed the crosses. MS performed the genotyping and analyzed the data. KS and MS wrote the manuscript. All authors read and approved the final manuscript.

\section{ACKNOWLEDGMENTS}

We would like to thank the Institute of Crop Science of the University of Hohenheim for providing the amaranth varieties (Baernkrafft, C6, Puerto Moutt and Pastewny) and the USDAARS for providing germplasm. This work was funded by an endowment of the Stifterverband der Deutschen Wissenschaft grant to KS (Project H1705701502016296 ). MS, LZ, AS, KK, and $\mathrm{MB}$ were supported by the Humboldt Reloaded project at the University of Hohenheim, Germany (BMBF 01PL11003).

\section{SUPPLEMENTARY MATERIAL}

The Supplementary Material for this article can be found online at: http://journal.frontiersin.org/article/10.3389/fpls.2016. 00816

Laser, K., and Lersten, N. (1972). Anatomy and cytology of microsporogenesis in cytoplasmic male sterile angiosperms. Botan. Rev. 38, 425-454.

Lehmann, J., Clark, R., and Frey, K. (1991). Biomass heterosis and combining ability in interspecific and intraspecific matings of grain amaranths. Crop Sci. 31, 1111-1116.

Maughan, P. J., Smith, S. M., Fairbanks, D. J., and Jellen, E. N. (2011). Development, characterization, and linkage mapping of single nucleotide polymorphisms in the grain amaranths (Amaranthus sp.). Plant Gen. J. 4, 92-101. doi: 10.3835/plantgenome2010.12.0027

Meyerowitz, E. M., and Pruitt, R. E. (1985). Arabidopsis thaliana and plant molecular genetics. Science 229, 1214-1218.

Moose, S. P., and Mumm, R. H. (2008). Molecular plant breeding as the foundation for 21st century crop improvement. Plant Physiol. 147, 969-977. doi: 10.1104/pp.108.118232

Mukasa, Y., Suzuki, T., and Honda, Y. (2007). Emasculation of Tartary buckwheat (Fagopyrum tataricum Gaertn.) using hot water. Euphytica 156, 319-326. doi: 10.1007/s10681-007-9377-7

Olsen, K. M., and Wendel, J. F. (2013). A bountiful harvest: genomic insights into crop domestication phenotypes. Ann. Rev. Plant Biol. 64, 47-70. doi: 10.1146/annurev-arplant-050312-120048 
Otsuka, J., Yamaguchi, S., Chigira, O., and Kato, K. (2010). Application of hot water emasculation to Acacia auriculiformis for controlled pollination. J. Forest Res. 15, 210-216. doi: 10.1007/s10310-009-0174-0

Peters, I., and Jain, S. (1987). Genetics of grain amaranths III. Gene-cytoplasmic male sterility. J. Heredity 78, 251-256.

Rastogi, A., and Shukla, S. (2013). Amaranth: a new millennium crop of nutraceutical values. Crit. Rev. Food Sci. Nutrit. 53, 109-125. doi: 10.1080/10408398.2010.517876

Reta Alemayehu, F., Bendevis, M. A., and Jacobsen, S.-E. (2015). The potential for utilizing the seed crop Amaranth (Amaranthus spp.) in east Africa as an alternative crop to support food security and climate change mitigation. J. Agron. Crop Sci. 201, 321-329. doi: 10.1111/jac.12108

Saghai-Maroof, M. A., Soliman, K. M., Jorgensen, R. A., and Allard, R. W. (1984). Ribosomal DNA spacer-length polymorphisms in barley: mendelian inheritance, chromosomal location, and population dynamics. Proc. Natl. Acad. Sci. U.S.A. 81, 8014-8018.

Sauer, J. (1967). The grain amaranths and their relatives: a revised taxonomic and geographic survey. Ann. Missouri Botan. Garden 54, 103-137.

Stetter, M. G., Müller, T., and Schmid, K. (2015). Incomplete domestication of South American grain amaranth (Amaranthus caudatus) from its wild relatives. bioRxiv.
Veerappan, V., Kadel, K., Alexis, N., Scott, A., Kryvoruchko, I., Sinharoy, S., et al. (2014). Keel petal incision: a simple and efficient method for genetic crossing in Medicago truncatula. Plant Methods 10:11. doi: 10.1186/17464811-10-11

Vega-Gálvez, A., Miranda, M., Vergara, J., Uribe, E., Puente, L., and Martínez, E. A. (2010). Nutrition facts and functional potential of quinoa (Chenopodium quinoa willd.), an ancient Andean grain: a review. J. Sci. Food Agricult. 90, 2541-2547. doi: 10.1002/jsfa. 4158

Conflict of Interest Statement: The authors declare that the research was conducted in the absence of any commercial or financial relationships that could be construed as a potential conflict of interest.

Copyright (c) 2016 Stetter, Zeitler, Steinhaus, Kroener, Biljecki and Schmid. This is an open-access article distributed under the terms of the Creative Commons Attribution License (CC BY). The use, distribution or reproduction in other forums is permitted, provided the original author(s) or licensor are credited and that the original publication in this journal is cited, in accordance with accepted academic practice. No use, distribution or reproduction is permitted which does not comply with these terms. 